\title{
PREDIÇÃO DA MASSA CORPORAL MAGRA DE ADULTOS BRASILEIROS ATRAVÉS DA ÁREA MUSCULAR DO BRAÇO
}

\author{
PREDICTION OF LEAN BODY MASS IN BRAZILIAN ADULTS THROUGH ARM MUSCLE AREA
}

PREDICCIÓN DE LA MASA CORPORAL MAGRA DE ADULTOS BRASILEÑOS A TRAVÉS DEL ÁREA

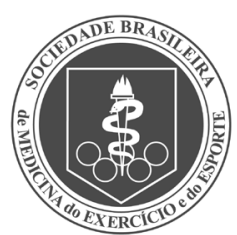

Artigo Original MUSCULAR DEL BRAZO

\begin{abstract}
Tane Kanope Ferreira Serpa' (Educador Fisico)

Fernando dos Santos Nogueira' (Educador Fisico)

Fernando Augusto Monteiro Saboia Pompeu' (Educador Fisico)

\section{Universidade Federal do Rio de Janeiro (UFRJ), Rio de Janeiro, RJ, Brasil.}

\section{Correspondência:}

Programa de Pós-graduação em Educação Física da Universidade Federal do Rio de Janeiro. Av. Carlos Chagas Filho 540, 21941-599, Rio de Janeiro, RJ, Brasil.tane.ufrj@gmail.com/ pompeu_fernando@hotmail.com/ nogueira_ufrj@hotmail.com

\section{RESUMO}

Introdução: A área muscular do braço $\left(A_{m b}\right)$ é componente da massa corporal magra (MCM). Objetivo: Propor uma equação para estimativa da MCM através da $A_{m b}$. Métodos: Quarenta e cinco voluntários masculinos, aparentemente saudáveis, com idade média de $22 \pm 3$ anos e massa corporal 74,9 \pm 8,43 kg, foram distribuídos randomicamente em grupos de validação interna (VI) e validação externa (VE). Tais sujeitos foram submetidos a medidas antropométricas e à hidrometria. Antes dos testes, os sujeitos receberam as seguintes orientações: a) evitar o consumo de cafeína e álcool nas 24 horas precedentes; b) não realizar atividade física extenuante ( $\geq 5 \mathrm{METS}$ ), pelo menos nas 12 horas prévias ao exame e; c) período pós-prandial de quatro horas. A área muscular do braço foi determinada através da equação: $A_{m a}=[C-(T \cdot \pi)]^{2} \div(4 \cdot \pi)$. Resultados: O modelo derivado para predição foi: $M C M=9,127+(0,625 \cdot M C)$ $+\left(0,139 \cdot A_{m b}\right) ; r^{2}=0,91, E P E=1,85 \mathrm{~kg}(2,5 \%$ da massa corporal ou MC). Para VE, não houve diferença significativa entre a MCM medida pela eletrobioimpedância e a prevista pela fórmula acima $(p=0,350)$, $r^{2}=0,94, C V \%=2,0 \%, C C l=0,97$ e $E P E=1,87$ kg. Conclusão: A equação de regressão múltipla derivada permite estimar a MCM de jovens brasileiros do sexo masculino.

Palavras-chave: composição corporal, impedância bioelétrica, área muscular do braço.

\begin{abstract}
Introduction: Arm muscle area $\left(A_{\text {ma }}\right)$ is a component of lean body mass (LBM). Objective: The objective of this study was to develop an equation for estimating LBM through the $A_{\text {ma. }}$ Methods: Forty-five apparently healthy young males, with an average age of $22 \pm 3$ years and body mass of $74.9 \pm 8.43 \mathrm{~kg}$, were randomly divided into two groups: internal validation (IV) and external validation (EV). The total sample was submitted to anthropometric and hydrometric measurements. Before the test, the subjects received the following instructions: a) to avoid caffeine and alcohol 24 hours before the test; $b$ ) not to do any strenuous physical activity ( $\geq 5 \mathrm{METS}$ ) for at least 12 hours prior to exam and; c) a postprandial period of 4 hours. $A_{m a}$ was obtained by the equation: $A_{m a}=[C-(T \cdot \pi)]^{2} \div(4 \cdot \pi)$. Results: The prediction model obtained was: $L B M=9.127+(0.625 \cdot B W)+\left(0.139 \cdot A_{m a}\right) ; r^{2}=0.91 ; S E E=1.85 \mathrm{~kg}(2.5 \% B W)$. For the $E V$, no significant difference was found between LBM measured by BIA and that provided by the formula $(p=0.35) ; r^{2}=0.94 ; C V \%=2.0 \% ; I C C=0.97$ and $S E E=1.87 \mathrm{~kg}$. Conclusion: The multiple regression equation enables the LBM to be estimated for young Brazilian males.
\end{abstract}

Keywords: body composition, bioelectrical impedance, arm muscle area.

\section{RESUMEN}

Introducción: El área muscular del brazo $\left(A_{m b}\right)$ es componente de la masa corporal magra (MCM). Objetivo: Proponer una ecuación para estimativa de la MCM a través de $A_{m b}$. Métodos: Cuarenta y cinco voluntarios masculinos, aparentemente saludables, con edad promedio de $22 \pm 3$ años y masa corporal 74,9 $\pm 8,43 \mathrm{~kg}$, fueron distribuidos aleatoriamente en grupos de validación interna (VI) y validación externa (VE). Tales sujetos fueron sometidos a medidas antropométricas y a hidrometría. Antes de los tests, los sujetos recibieron las siguientes orientaciones: a) evitar el consumo de cafeína y alcohol en las 24 horas precedentes; b) no realizar actividad física extenuante ( $\geq 5$ METs), por lo menos en las 12 horas previas al examen y, c) período postprandial de cuatro horas. El área muscular del brazo fue determinada a través de la ecuación: $A_{m a}=[C-(T \cdot \pi)]^{2} \div(4 \cdot \pi)$. Resultados: El modelo derivado para predicción fue: $M C M=9,127+(0,625 \cdot M C)+\left(0,139 \cdot A_{m b}\right) ; r^{2}=0,91, E P E=$ $1,85 \mathrm{~kg}$ (2,5\% de la masa corporal o MC). Para VE, no hubo diferencia significativa entre la MCM medida por la electrobioimpedancia y la prevista por la fórmula de arriba $(p=0,350), r^{2}=0,94, C V \%=2,0 \%, C C l=0,97$ y EPE= 1,87 kg. Conclusión: La ecuación de regresión múltiple derivada permite estimar la MCM de jóvenes brasileños del sexo masculino.

Palabras clave: composición corporal, impedancia bioeléctrica, área muscular del brazo. 


\section{INTRODUÇÃO}

A análise da composição corporal possibilita a estratificação do risco fisiopatológico associado à adiposidade corporal. Tal exame, também, fornece informações relacionadas às mudanças na composição corporal para orientar dietas nutricionais e a prescrição de exercícios ${ }^{1}$. Diversas técnicas para a inferência da composição corporal já foram descritas², entretanto, estas quantificações dependem de procedimentos laboratoriais complexos e de alto custo. Há, portanto, necessidade do desenvolvimento de técnicas mais simples, menos dispendiosas e com boa precisão para as populações brasileiras ${ }^{3}$.

Dentre as técnicas laboratoriais, a pesagem hidrostática destaca-se pela ampla utilização para a validação de outros métodos ${ }^{4}$. Nessa técnica assume-se que o corpo é composto pelos compartimentos da massa de gordura e o da massa corporal magra (MCM). A inferência da densidade corporal possibilita a estimava daqueles componentes da composição corporal5. Trata-se, no entanto, de uma técnica de execução complexa e de custo elevado, portanto, limitada ao ambiente laboratorial ${ }^{6}$.

Outra técnica laboratorial muito utilizada é a eletrobioimpedância tetrapolar (BIA $)^{7}$. A validade e a confiabilidade da BIA foram determinadas através de estudos que a confrontaram com a pesagem hidrostática. Os métodos apresentaram boa associação, com elevados coeficientes de correlação para a estimativa da MCM ( $r=0,84$ a 0,98), e reduzidos erros padrões da estimativa $(E P E=1,31 \text { a } 5,8 \mathrm{~kg})^{7-13}$. A última técnica, no entanto, não prescinde de examinadores qualificados e de uma longa fase de preparação a ser criteriosamente seguida pelo avaliado ${ }^{14}$.

A antropometria, por outro lado, pode ser uma boa alternativa para inferência da composição corporal ${ }^{15}$. A técnica antropométrica da estimativa da área muscular e do anel de gordura do braço ${ }^{16}$ é uma boa opção para medidas de campo. O último método, além de ter um reduzido tempo de aplicação e custo operacional, parece ter boa aplicabilidade na avaliação da composição corporal.

Considerando a aplicabilidade da estimativa da área muscular e a exiguidade de estudos com populações brasileiras ${ }^{3}$, o objetivo da presente investigação foi desenvolver um modelo matemático válido e acurado para inferência da MCM, de sujeitos jovens adultos masculinos.

\section{MÉTODOS}

Foram recrutados para a presente investigação 45 voluntários masculinos aparentemente saudáveis, assintomáticos e praticantes regulares de exercícios físicos (tabela 1). Os sujeitos foram divididos, através de randomização, em dois grupos. O tamanho da amostra foi estabelecido conforme sugerido por Hopkins ${ }^{17}$. Trinta voluntários foram sorteados para o grupo de validação interna (VI) e 15 para o grupo de validação externa (VE).

Antes do teste os sujeitos receberam a orientação de absterem-se tanto de esforços físicos extenuantes ( $\geq 5 \mathrm{METs}$ ), como de cafeína e álcool nas 24 horas precedentes. Recomendou-se, também, o período pós-prandial de pelo menos quatro horas, de não fazer uso de medicamentos diuréticos e, finalmente, esvaziar a bexiga 30 minutos antes das medidas ${ }^{18,19}$. Todos os procedimentos aqui adotados foram aprovados

Tabela 1. Características antropométricas.

\begin{tabular}{c|c|c|c}
\hline Variáveis & VI $(\mathbf{n = 3 0})$ & VE $(\mathbf{n = 1 5 )}$ & $\mathbf{P}$ \\
\hline Idade $(\mathrm{anos})$ & $23 \pm 3$ & $21 \pm 2$ & 0,067 \\
\hline Massa $(\mathrm{kg})$ & $75,1 \pm 7,9$ & $71,9 \pm 9,4$ & 0,261 \\
\hline Estatura $(\mathrm{cm})$ & $176,6 \pm 6,4$ & $174,5 \pm 5,9$ & 0,292 \\
\hline MCM $(\mathrm{BIA}, \mathrm{kg})$ & $65,3 \pm 6,5$ & $62,4 \pm 7,3$ & 0,212 \\
\hline $\mathrm{A}_{\text {mb }}\left(\mathrm{cm}^{2}\right)$ & $66,2 \pm 13,75$ & $63,4 \pm 15$ & 0,540 \\
\hline n número de voluntários; V: grupo de validade interna; VE: grupo de validade externa; : significância da diferença
\end{tabular}

pelo Comitê de Ética para Experimentos com Seres Humanos (CAE 09237613.1.0000.5257). Os participantes do estudo foram informados sobre os procedimentos a serem realizados e assinaram um termo de consentimento livre e esclarecido.

\section{Procedimentos experimentais}

Os sujeitos realizaram visitas ao laboratório, no mesmo período do dia. A visita ao laboratório destinava-se a determinação da composição corporal através da antropometria, com medidas de massa corporal (MC), estatura, diâmetros, perímetros e dobras cutâneas (DC) ${ }^{20}$ e da hidrometria. Uma segunda visita foi realizada por 30 sujeitos, em um intervalo de tempo de $24 \mathrm{~h}$ a sete dias, para a determinação da confiabilidade do método.

Para coleta das dobras cutâneas, utilizou-se um plicômetro com pressão nas hastes de 10,0 $\mathrm{g} / \mathrm{mm}^{2}$ e escala de 1,0 mm (Lange Skinfold Caliper $^{\circledR}$, Beta Technology ${ }^{\circledR}$, Santa Cruz, CA, EUA). Os diâmetros e perímetros foram medidos através de um paquímetro antropométrico e uma trena metálica, com escala de 0,1 cm (Samy American Medical ${ }^{\circledR}$, São Bernardo do Campo, SP, Brasil). A massa corporal e a estatura foram medidas através de uma balança mecânica com estadiômetro, com resolução de 0,1 kg e 0,1 cm, respectivamente (Welmy ${ }^{\circledR}$, Santa Bárbara d'Oeste, SP, Brasil). O conteúdo de água corporal foi determinado através de eletrobioimpedância tetrapolar, com amplitude de resistência de 200-1500 Ohms, resolução em um Ohm, precisão de 1\% e intensidade e frequência de corrente elétrica de $800 \mu \mathrm{A}$ e $50 \mathrm{kHz}$, respectivamente (BIA 310e Bioimpedance Analyzer ${ }^{\circledast}$, Biodynamics ${ }^{\circledR}$, Seattle, WA, EUA).

A área muscular do braço $\left(A_{m b}\right)$ foi obtida conforme o sugerido por Frisancho ${ }^{16}$.

$$
A_{m b}=\frac{[C-(T \cdot \pi)]^{2}}{(4 \cdot \pi)}
$$

Onde C é a circunferência de braço direito relaxado, no ponto mesoumeral e Té a DC de tríceps.

\section{Análise estatística}

O tratamento estatístico foi realizado através dos aplicativos Statistical Package for the Social Sciences ${ }^{\circledR}$ (SPSS, EUA) e Microsoft Excel ${ }^{\circledR}$ para Microsoft Windows $7^{\circledR}$ (Microsoft, EUA). Empregou-se a estatística descritiva com a média \pm desvio padrão (DP). A precisão das medidas antropométricas do avaliador foi calculada através do erro técnico de medida (ETM), proposto por Norton e Olds ${ }^{21}$.

O ETM intra-avaliador foi calculado através das formulas:

\begin{tabular}{|c|c|}
\hline ETM ${ }_{\text {absoluto }}=$ & $\frac{\sqrt{\Sigma} d^{2}}{2}$ \\
\hline ETM $M_{\text {relativo }}=$ & $\frac{E T M_{\text {absoluto }} \cdot 100}{V M V}$ \\
\hline
\end{tabular}

Onde: $\Sigma \mathrm{d}^{2}=$ soma dos desvios ao quadrado; $\mathrm{N}=$ número de indivíduos avaliados; VMV = Valor médio da variável.

Previamente à determinação da equação de regressão múltipla, uma matriz de correlação foi obtida visando verificar as variáveis que melhor discriminavam a MCM.

Os limites de concordância de Bland-Altman (B-A) 22 foram inferidos entre os resultados obtidos pela BIA e os calculados pelo modelo de predição. $\mathrm{O}$ grau de associação entre os valores medidos e preditos foi determinado pelo coeficiente de correlação intraclasse (CCI ${ }^{23}$. 
O erro da predição também foi observado por meio do erro padrão da estimativa (EPE) e do coeficiente de variação (CV \%). Todos os testes estatísticos foram realizados no nível de significância $p \leq 0,05$.

\section{RESULTADOS}

As características antropométricas dos voluntários encontram-se na tabela 1. A massa corporal, $A_{m b}$, circunferência do braço relaxado e a circunferência do braço contraído foram as variáveis que apresentaram as melhores correlações com a MCM, ou $r=0,922, r=0,71, r=0,76$ e r $=0,78$ (tabela 2) e o ETM encontrado foi de até $5 \%$ para DC e até $1 \%$ para as outras medidas ${ }^{21}$.

Os modelos para predição da MCM gerados através do grupo de VI estão representados na tabela 3.

No grupo VE, não foram observadas diferenças significativas entre os três modelos e a BIA. Entretanto, foi observado que a equação 3 apresentou valores mais elevados do coeficiente de determinação $\left(R^{2}=0,91\right)$ e da correlação intraclasse $(C \mathrm{Cl}=0,97) \mathrm{e}$, menor de erro $(\mathrm{EPE}=2,0 \mathrm{~kg})$.

Na figura 1 observa-se a validade externa, obtida para o grupo VE, e os limites de concordância para $\pm 1,96$ DP.

\section{DISCUSSÃO}

No presente estudo foi proposto um modelo para a predição da MCM de jovens adultos brasileiros masculinos, através de parâmetros antropométricos. Os resultados aqui descritos demonstram que não houve diferença significativa entre as medidas da MCM através da BIA e do modelo matemático. Até o presente momento, não foram localizados nas bases Web of Science, Medline e SciELO, estudos semelhantes para população brasileira. Isso dificultou a análise e discussão dos dados aqui apresentados.

A inferência da composição corporal através da BIA baseia-se no comportamento de uma corrente elétrica durante a passagem por tecidos biológicos. Considerando, portanto, que a MCM é mais hidratada e possui maior quantidade de eletrólitos do que no tecido adiposo, maior MCM produzirá maior condutibilidade e menor impedância ao fluxo da corrente $^{24,25}$. Essa técnica apresenta excelente validade e confiabilidade quando confrontado com a técnica de densitometria (tabela 4),25-28.

As variáveis com maior correlação com a MCM foram a massa corporal, $A_{m b}$ e as circunferências de braço relaxado e contraído (tabela 2). Entretanto, quando as variáveis de circunferência foram utilizadas para a predição da MCM, o modelo matemático apresentou menor coeficiente de determinação e maior erro padrão da estimativa para circunferência de braço relaxado e circunferência de braço contraído $\left(R^{2}=0,86\right.$; $E P E=$ 2,4 $\mathrm{kg} \mathrm{e} \mathrm{R}^{2}=0,87$; $E P E=2,3$; respectivamente).

Apesar de não haver diferenças significativas entre os modelos
Tabela 3. Modelos para predição da massa corporal magra.

\begin{tabular}{c|c|c|c|c|c}
\hline Varáveis Antropométricas & $\begin{array}{c}\text { Equação de } \\
\text { Regressão }\end{array}$ & Modelo No & $\mathbf{R}^{\mathbf{2}}$ & $\mathbf{E P E}$ & $\mathbf{C C l}$ \\
\hline Massa; C. braço relaxado & $\mathrm{MCM}=$ & 1 & 0,86 & 2,43 & 0,95 \\
\hline Massa; C. braço contraído & $\mathrm{MCM})$ & 2 & 0,87 & 2,34 & 0,96 \\
\hline Massa; $\mathrm{A}_{\mathrm{mb}}$ & $\mathrm{MCM}=$ & 3 & 0,91 & 2,0 & 0,97 \\
\hline
\end{tabular}

$\mathrm{A}_{\mathrm{mb}}$ : Área muscular do braço; C: Circunferência; DC: Dobra Cutânea; MCM: Massa Corporal Magra; $\mathrm{R}^{2}$ : Coeficiente de determinaçăo; EPE: Erro padrão da estimativa e CCl: Coeficiente de correlação intraclasse.
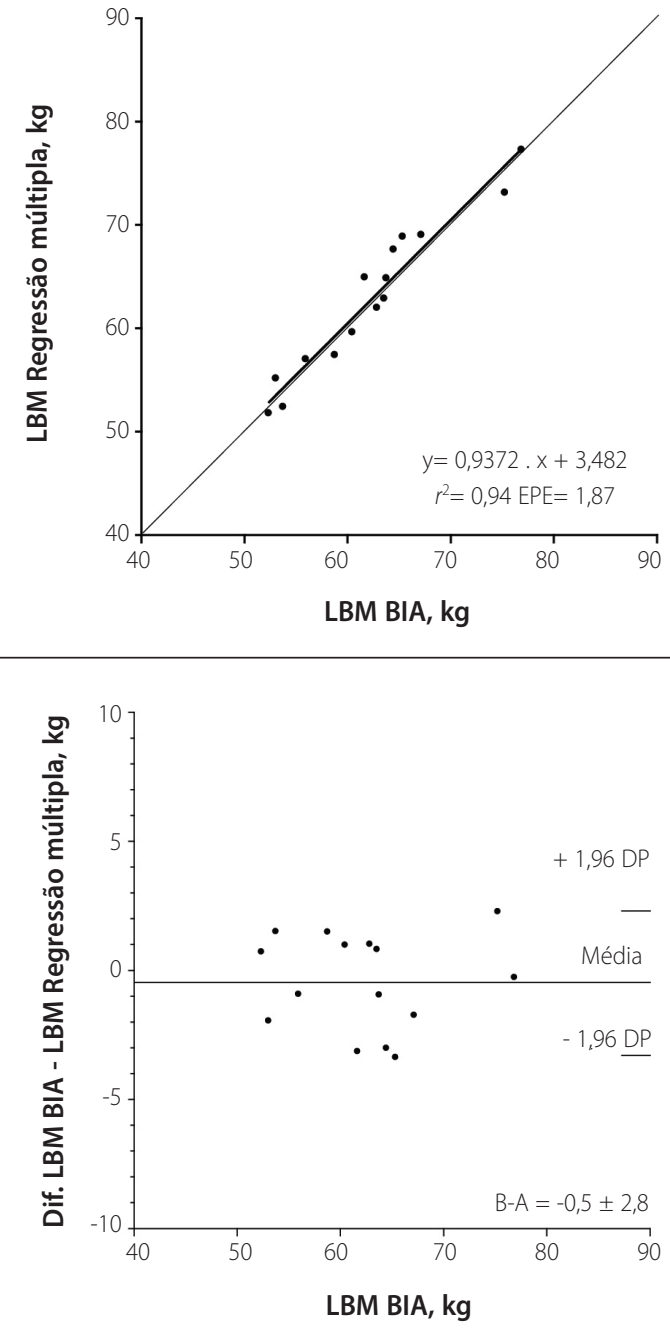

Figura 1. Correlação e limites de concordância entre os valores de MCM obtidos no modelo de regressão e BIA para o Grupo VE.

Tabela 2. Matriz de correlação para dados antropométricos.

\begin{tabular}{|c|c|c|c|c|c|c|c|c|}
\hline Variáveis & Idade (anos) & Estatura $(\mathrm{cm})$ & Massa $(\mathrm{kg})$ & $A_{m b}\left(\mathrm{~cm}^{2}\right)$ & C. braço relaxado $(\mathrm{cm})$ & C. braço contraido $(\mathrm{cm})$ & DC tríceps $(\mathrm{mm})$ & MCM (kg) \\
\hline Idade (anos) & 1 & $-0,02$ & 0,15 & 0,27 & 0,24 & 0,34 & $-0,08$ & 0,12 \\
\hline Estatura $(\mathrm{cm})$ & & 1 & $0,56^{* *}$ & $0,55^{* *}$ & 0,08 & 0,17 & 0,05 & $0,55^{* *}$ \\
\hline Massa (kg) & & & 1 & $0,63^{* *}$ & $0,72^{* *}$ & $0,72^{* *}$ & 0,39 & $0,92^{* *}$ \\
\hline $\mathrm{A}_{\mathrm{mb}}\left(\mathrm{cm}^{2}\right)$ & & & & 1 & $0,92^{* *}$ & $0,93^{* *}$ & $-0,21$ & $0,72^{* *}$ \\
\hline C. braço relaxado $(\mathrm{cm})$ & & & & & 1 & $0,97^{* *}$ & 0,193 & $0,76^{* *}$ \\
\hline C. braço contraído $(\mathrm{cm})$ & & & & & & 1 & 0,08 & $0,78^{* *}$ \\
\hline DC tríceps (mm) & & & & & & & 1 & 0,09 \\
\hline MCM (kg) & & & & & & & & 1 \\
\hline
\end{tabular}


Tabela 4. Validade e confiabilidade da Eletrobioimpedância tetrapolar.

\begin{tabular}{|c|c|c|c|}
\hline Autores & Ano & Faixa etária & $r^{2} ;$ EPE \\
\hline Lukaski et al. ${ }^{7}$ & 1986 & $18-50$ & 0,$98 ; 2,5$ \\
\hline Lukaski et al. ${ }^{26}$ & 1985 & $19-42$ & 0,$98 ; 2,6$ \\
\hline Segal et al. ${ }^{28}$ & 1988 & $17-62$ & 0,$94 ; 2,84$ \\
\hline Segal et al. ${ }^{27}$ & 1985 & $17-59$ & 0,$96 ; 3,06$ \\
\hline Carvalho \& Neto ${ }^{25}$ & 1999 & $18-30$ & 0,$95 ; * * *$ \\
\hline
\end{tabular}

matemáticos confrontados através da transformação de Fisher, a equação 3 aproxima-se mais do valor hidromético da MCM. Esta equação é também vantajosa por utilizar o cálculo da área muscular do braço, índice esse que fornece outras informações relevantes, como a estimativa da força voluntária máxima dos membros superiores e tronco ${ }^{3}$.

A proporção entre os tecidos corporais é alterada com a idade ${ }^{16}$. Esperava-se, portanto, uma forte correlação entre idade e MCM ${ }^{29,30}$, porém, na presente investigação, esta variável não contribuiu para melhorar a relação entre $A_{m b}$ e a MCM. Explica-se este resultado pela estreita variação na faixa etária dos sujeitos desta investigação. Esta va- riável, também, não apresentou distribuição gaussiana, testada através da estatística de Shapiro-Wilk $(p=0,006)$.

\section{CONCLUSÃO}

À partir dos dados encontrados no presente estudo, pode-se sugerir que existe excelente correlação entre os dados referentes à MCM aferidos pela BIA e pelo modelo matemático para jovens adultos brasileiros do sexo masculino. Tendo em vista que as equações para predição são específicas para as diferentes populações ${ }^{30}$, sugere-se a realização de estudos semelhantes com indivíduos do sexo feminino e de outras faixas etárias.

\section{AGRADECIMENTOS}

Os autores agradecem à Fundação de Amparo à Pesquisa Carlos Chagas Filho do Estado do Rio de Janeiro, Brasil, pelo apoio financeiro recebido.

Todos os autores declararam não haver qualquer potencial conflito de interesses referente a este artigo.

\section{REFERÊNCIAS}

1. Rodrigues MN, Silva SC, Monteiro WD, Farinatti PT. Estimativa da gordura corporal através de equipamentos de bioimpedância, dobras cutâneas e pesagem hidrostática. Rev Bras Med Esporte. 2001;7(4):125-31.

2. Forslund AH, Johansson AG, Sjodin A, Bryding G, Ljunghall S, Hambraeus L. Evaluation of modified multicompartment models to calculate body composition in healthy males. Am J Clin Nutr. 1996;63(6):856-62

3. Pompeu FA, Gabriel D, Pena BG, Ribeiro P. Áreas de secção transversa do braço: implicações técnicas e aplicações para avaliação da composição corporal. Rev Bras Med Esporte. 2004;10(3):202-6.

4. Pollock ML, Hickman T, Kendrick Z, Jackson A, Linnerud AC, Dawson G. Prediction of body density in young and middle-aged men. J Appl Physiol. 1976;40(3):300-4.

5. Siri WE. Body composition from fluid spaces and density. In: Brozek J, Henschel A, editors. Techniques for measuring body composition. Washington DC: National Academy of Science; 1961

6. Lukaski HC. Methods for the assessment of human body composition: traditional and new. Am J Clin Nutr. 1987;46(4):537-56.

7. Lukaski HC, BolonchukWW, Hall CB, Siders WA. Validation of tetrapolar bioelectrical impedance method to assess human body composition. J Appl Physiol. 1986;60(4):1327-32

8. Conlisk EA, Haas JD, Martinez EJ, Flores R, Rivera JD, Martorell R. Predicting body composition from anthropometry and bioimpedance in marginally undernourished adolescents and young adults. Am J Clin Nutr. 1992;55(6):1051-60

9. Deurenberg P, Kusters CS, Smit HE. Assessment of body composition by bioelectrical impedance in children and young adults is strongly age-dependent. Eur J Clin Nutr. 1990;44(4):261-8.

10. Dittmar M, Reber H. New equations for estimating body cell mass from bioimpedance parallel models in healthy older Germans. Am J Physiol Endocrinol Metab. 2001;281(5):E1005-14.

11. Kyle UG, Genton L, Slosman DO, Pichard C. Fat-free and fat mass percentiles in 5225 healthy subjects aged 15 to 98 years. Nutrition. [Research Support, Non-U.S. Gov't]. 2001;17(7-8):534-41.

12. Segal KR, Burastero S, Chun A, Coronel P, Pierson RN, Jr., Wang J. Estimation of extracellular and total body water by multiple-frequency bioelectrical-impedance measurement. Am J Clin Nutr. 1991;54(1):26-9.

13. Sun SS, Chumlea WC, Heymsfield SB, Lukaski HC, Schoeller D, Friedl K, et al. Development of bioelectrical impedance analysis prediction equations for body composition with the use of a multicomponent model for use in epidemiologic surveys. Am J Clin Nutr. 2003;77(2):331-40.

14. Wilmore JH, Costill DL, Kenney WL. Fisiologia do Esporte e do Exercício. São Paulo: Manole; 2010.
15. Martins KA, Monego ET, Paulinelli RR, Freitas-Junior R. Comparison of methods to evaluate total body fat and its distribution. Rev Bras Epidemiol. 2011;14(4):677-87.

16. Frisancho AR. Triceps skin fold and upper arm muscle size norms for assessment of nutrition status. Am J Clin Nutr. 1974:27(10):1052-8.

17. Hopkins WG. A New View of Statistics. 2012 [2012]; Available from: http://sportsci.org/resourse/stats

18. Tanaka NI, Miyatani M, Masuo Y, Fukunaga T, Kanehisa H. Applicability of a segmental bioelectrical impedance analysis for predicting the whole body skeletal muscle volume. J Appl Physiol. 2007;103(5):1688-95.

19. Guedes DP. Procedimentos clínicos utilizados para análise da composição corporal. Rev Bras Cineantropom Desemp Humano.2013;15(1):113-29.

20. Pollock ML, Wilmore JH. Exercício na saúde e na doença. Avaliação e prescrição para prevenção e reabilitação. 2ed. ed. Rio de Janeiro: MEDSI; 1993.

21. Norton K, Olds T. Antropométrica. Porto Alegre: Artmed; 2005.

22. Bland JM, Altman DG. Statistical methods for assessing agreement between two methods of clinical measurement. Lancet. 1986;1(8476):307-10.

23. Bartko JJ. The intraclass correlation coefficient as a measure of reliability. Psychol Rep. 1966;19(1):3-11.

24. Glaner MF. Validação cruzada de equações de impedância bioelétrica em homens. Rev Bras Cineantropom Desemp Humano. 2005;7(1):5-11.

25. Carvalho ABR, Neto CSP. Composição corporal através dos métodos da pesagem hidrostática e impedância bioelétrica em universitários. Rev Bras Cineantropom Desemp Humano. 1999;1 (1):18-23.

26. Lukaski HC, Johnson PE, Bolonchuk WW, Lykken Gl. Assessment of fat-free mass using bioelectrical impedance measurements of the human body. Am J Clin Nutr. 1985;41(4):810-7.

27. Segal KR, Gutin B, Presta E, Wang J, Van Itallie TB. Estimation of human body composition by electrical impedance methods: a comparative study. J Appl Physiol. 1985;58(5):1565-71.

28. Segal KR, Van Loan M, Fitzgerald PI, Hodgdon JA, Van Itallie TB. Lean body mass estimation by bioelectrical impedance analysis: a four-site cross-validation study. Am J Clin Nutr. 1988;47(1):7-14.

29. Houtkooper LB, Going SB, Lohman TG, Roche AF, Van Loan M. Bioelectrical impedance estimation of fat-free body mass in children and youth: a cross-validation study. J Appl Physiol. 1992;72(1):366-73.

30. Rech CR, Salomons E, Lima LRA, Petroski EL, Glaner MF. Estimativa da Massa Muscular esquelética em mulheres idosas: validade da impedância bioelétrica. Rev Bras de Med Esporte. 2010;16(2):95-8. 